\section{Properties and prediction accuracy of a sigmoid function of time-determinate growth}

\author{
Róbert Sedmák ${ }^{(1-2)}$, Lubomír Scheer ${ }^{(1)}$
}

The properties and short-term prediction accuracy of a mathematical model of sigmoid time-determinate growth, denoted as "KM-function", are presented. The model is suitable for representing the asymmetrical sigmoid growth of an organism, starting at zero size and terminating when the final size is reached. The function assumes a finite length of the growth period and includes a parameter interpretable as the expected lifespan of the organism. Moreover, the possibility for growth curve inflexion at any age allows to model S-shaped growth trajectories with various degree of asymmetry. The suitability of such theoretical model to predict the real growth of trees was empirically assessed. Three and four-parameters forms of the KM-function was compared with three classical (Richards, Korf and Weibull) growth functions using two parametrization methods, i.e., the nonlinear least squares (NLS) and the Bayesian method. The parametrization/validation dataset was made of 67 tree diameter series obtained from stem analyses. Main results may be summarized as follows: (i) the use of the three-parameter KM-function with the Bayes parametrization method is recommended when the minimization of prediction bias is required; (ii) the best short-term prediction results, in terms of minimization of root square error (RMSE), were obtained using the four-parameter Weibull's function and the NLS parametrization method; (iii) three-parameter functions parametrized by Bayesian methods show a considerably smaller RMSE (by 15$25 \%$ ) and biases (by $40-60 \%$ ) than four-parameter functions using the NLS method. Overall, our results confirmed the relative usefulness of the KM-function in comparison with classical growth functions, especially when combined with Bayesian parametrization methods.

Keywords: Growth Function, Determinate Growth, Nonlinear Least Squares, Bayes, Prediction

\section{Introduction}

Changes in plant size over the lifespan are typically characterized by an S-shaped growth trajectory (Pretzsch 2009, Weiskittel et al. 2011, Zeide 1993). Growth functions represent simple, non-linear equations describing the sigmoidal change in size of an individual or population with time. Their mathematical forms are derived empirically or conceived from the biological knowledge of the growth of living organisms (Burkhart \& Tomé 2012). Historically, the Gompertz, logistic, Mitscherlich or Korf's growth mod-

els rank among the most successful functions used in modeling the plant growth (Gompertz 1825, Korf 1939, Mitscherlich 1919, Verhulst 1838). Currently, the most used growth model is the Richards' function (Richards 1959) which was obtained by the generalization of the Bertallanffy's growth function (Bertalanffy 1957). Among the growth functions with unique properties, the Weibull's or Sloboda's equations and the increasingly popular Schnute's growth function are also worth to be mentioned (Weibull 1951, Schnute 1981, Sloboda 1971). A com-

(1) Faculty of Forestry, Technical University in Zvolen, T.G. Masaryka 24, 96053 Zvolen (Slovak Republic); (2) Faculty of Forestry and Wood Sciences, Czech University of Life Sciences Prague, Kamýcká 1176, 16521 Praha 6 - Suchdol (Czech Republic)

@ Lubomír Scheer (scheer@tuzvo.sk)

Received: Jan 15, 2014 - Accepted: Oct 17, 2014

Citation: Sedmák R, Scheer L, 2015. Properties and prediction accuracy of a sigmoid function of time-determinate growth. iForest 8: 631-637 [online 2015-01-13] URL: http://www. sisef.it/iforest/contents/?id=ifor1243-007

Communicated by: Renzo Motta prehensive survey of the historical background, the mathematical and statistical properties of the mentioned growth functions may be found in the works by Zeide (1993), Seber \& Wild (2003), Tsoularis \& Wallace (2002) and Karkach (2006).

The application of growth functions is now more common than ever. Growth functions are based on relatively simple equations with a limited number of parameters; nonetheless, they show a good ability to encompass complicated growth processes (Zeide 2004). A direct prediction of the integral quantities of growth at individual or population level by means of simpler growth functions can be more precise than prediction from the sum of the individual components of growth (cells, tissues and organs) obtained by more complicated process models. In general, this can be considered a major paradox of ecological modeling and represents a considerable challenge to plant ecology science (Pretzsch 2009, Seber \& Wild 2003, Zeide 2003).

From a mathematical point of view, each growth function suitable for the description of sigmoid growth must be able to describe a nonlinear, monotonically increasing growth pattern having a concave shape along the early life stages. At later stages, this turns into a convex function which approaches a final asymptote. For these reasons, growth functions are characterized by the inclusion of three or four parameters defining the position and shape of the growth curve (Fekedulegn et al. 1999, Fitzhugh 1976). In gene$\mathrm{ral}$, the asymptotic parameter $\beta_{0}$ is present in all classical growth functions (Birch 1999), and represents the limit (eqn. 1):

$$
\beta_{0}=\lim _{t \rightarrow \infty} \int_{0}^{t} f^{\prime}(t)
$$

Hence, $\beta_{0}$ is the asymptotic value which the growth function approaches towards the end of an organism's life, and which the growth function will reach after an infinite growth period.

A detailed analysis on the mathematical structure of some important growth functions by Shvets \& Zeide (1996) reported that even after 200 years of intensive research, there is still opportunity for improvement of the mathematical structure of existing growth functions. Karkach (2006) distinguishes two general growth types: determinate and non-determinate growth. The former applies when the growth of an organism terminates at a certain development stage or at a final (maximum) size, whereas non-determinate growth continues throughout the organism's lifespan, regardless of the degree of physiological maturity attained. Due to the existence of the asymptotic parameter $\beta_{0}$, all classical growth functions are non-determinate growth models that implicitly entail an infinite length of the growth period. This 
Tab. 1 - List of the growth models compared in this study.

\begin{tabular}{lll}
\hline Model (abbr) & Integral form & Source \\
\hline KM-function (KM3) & $y=y_{\max }\left[1-\left(1-(t / 500)^{\mathrm{b}}\right)^{\mathrm{c}}\right]$ & Kumaraswamy (1980) \\
KM-function (KM4) & $y=y_{\max }\left[1-\left(1-\left(t / t_{\max }\right)^{\mathrm{b}}\right)^{\mathrm{c}}\right]$ & Kumaraswamy (1980) \\
Richards function (R3) & $y=y_{\max }\left(1-e^{-\mathrm{bt}}\right)^{\mathrm{c}}$ & Zeide (1993) \\
Richards function (R4) & $y=y_{\max }\left(1-d e^{-\mathrm{bt} t}\right)^{\mathrm{c}}$ & Richards (1959), Fitzhugh (1976) \\
Korf function (KF3) & $y=y_{\max } e^{(-\mathrm{bt})^{\wedge} \mathrm{c}}$ & Korf (1939), Li et al. (2000) \\
Weibull function (W4) & $y=a-d e^{-(\mathrm{bt})^{\wedge} \mathrm{c}}$ & Weibull (1951), Ratkowsky (1983) \\
\hline
\end{tabular}

fact is clearly not consistent with the biological reality. The use of an asymptotic growth function for a finite life length of every living organism is possible only because they predict very small, practically negligible growth rates at the oldest ages.

Recently, two attempts to solve the aforementioned issue has been reported in the literature. Yin et al. (2003) proposed a new flexibile empirical function of sigmoid determinate growth, obtained by modifying the probability density function of the beta distribution. Sedmák \& Scheer (2012) derived another sigmoid function of determinate growth, called KM-function, based on a modification of the Kumaraswamy's distribution function (Kumaraswamy 1980). Generally, both probability distributions are suitable for double-bounded random variables, and consequently they are theoretically wellsuited for the description of determinate growth within a finite period of time.

Growth functions are used as semi-empirical models whose parameters are inferred from empirical measurements of the growth of an organism (Karkach 2006, Shvets \& Zeide 1996, Zeide 2004). Their parameter

estimations are carried out by means of different theoretical methods, mainly based on non-linear regressions. A wide range of methods of classic (Fisherian) frequentist statistics (Seber \& Wild 2003) or the alternative Bayesian statistics (Carlin \& Louis 2000) are currently used to this purpose.

To estimate the parameters in the regression models, the most common methods used by the frequentist school are the maximum likelihood estimation (MLE) and the ordinary least squares (OLS). The OLS method is derived from the MLE by introducing the simplified assumptions of normality, variance homogeneity and uncorrelatedness of residual deviations of the regression model. Using the OLS method, parameter estimation is almost exclusively related to the solution of a set of non-linear equations by numerical techniques. In such cases, the OLS method is also called nonlinear least squares (NLS) estimation.

An alternative method for the estimation of growth function parameters is based on a Bayesian approach. The basic difference between Fisherian and Bayesian statistical schools relies on a different understanding of

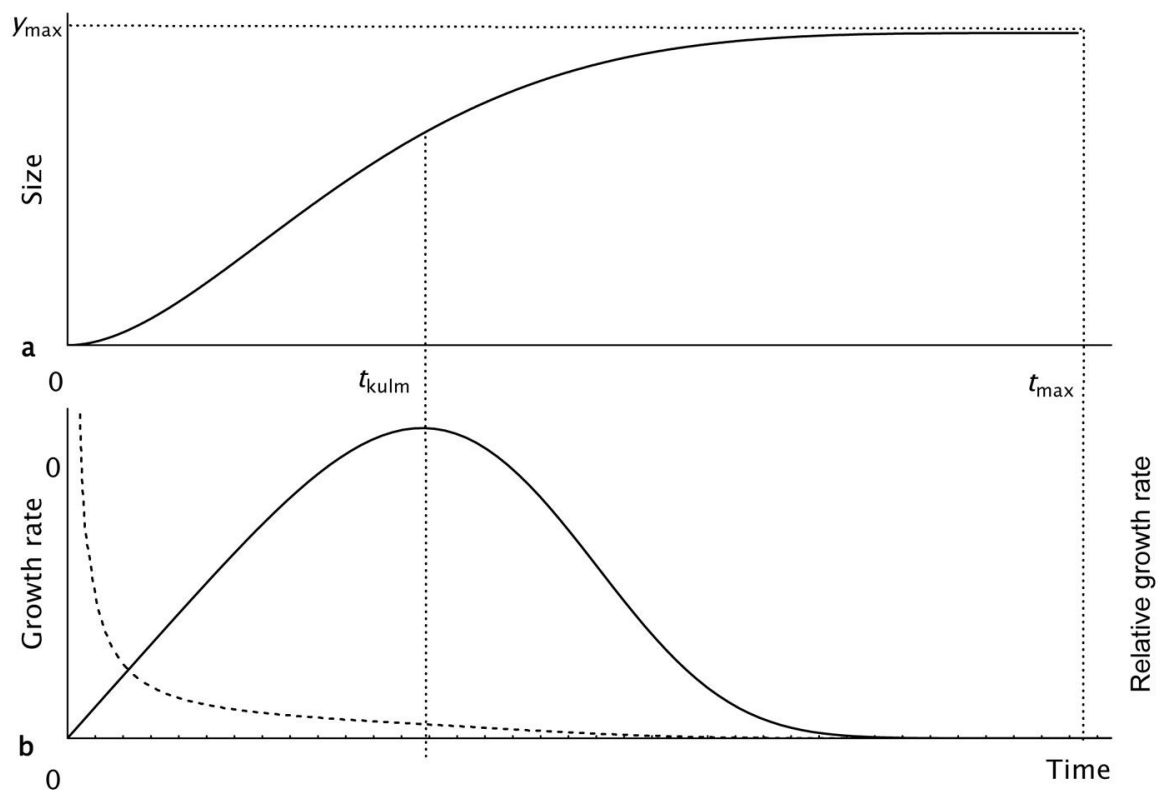

Fig. 1 - Growth trajectory described by the sigmoid KM-function. (a): Growth period starts at $t=0$ and ends at the age $t_{\max }$, at which the exact final value is reached; (b): full and broken lines show the corresponding courses of growth and relative growth rates, which are exactly equal to zero at the age $t_{\max }$. the probability concept (D'Agostini 2003). In the Bayesian estimation, the a priori joint probability distribution of possible parameter values in the model is combined with information from data represented by the likelihood function.

The objective of this study was to assess the performances of the KM-function in modeling the sigmoid determinate growth in living organisms and to analyse its theoretical properties. The analysis was carried out by comparing the properties and prediction accuracy of the KM-function with those of selected classical growth functions. The statistical properties of growth functions were compared based on empirical data, made by diameter growth series of individual trees obtained from stem analyses. Two methods of statistical parametrization were used: the classic nonlinear least squares method, and the Bayesian parametrization method based on the Markov chain Monte Carlo procedure.

\section{Material and methods}

\section{Growth functions included into comparison}

An overview of the growth functions used in this study is reported in Tab. 1. The first function was a time-determinate KM-function with the following integral form (eqn. 2):

$$
y=y_{\max }\left[1-\left(1-\left(\frac{t}{t_{\max }}\right)^{b}\right)^{c}\right]
$$

where $0 \leq t \leq t_{\max }, t_{\max }>0, y_{\max }>0, \mathrm{~b}>0, \mathrm{c}>$ 0 . The parameter $y_{\max }$ is interpreted as the final (maximum) size of an organism attained at the age $t_{\max }$. If the parameter $t_{\max }$ is fixed on the basis of a clear biological interpretation, the new function will have only three parameters. The parameters $b$ and $c$ determine the position of its inflexion point within the life cycle. The growth rate equation can be obtained by derivation from eqn. 2 as follows (eqn. 3):

$$
\frac{d y}{d t}=y_{\max } b c t_{\max }^{-1}\left(\frac{t}{t_{\max }}\right)^{b-1}\left[1-\left(\frac{t}{t_{\max }}\right)^{b}\right]^{c-1}
$$

A typical course of the KM-function over time and that of the derived absolute and relative growth rates $(R G R)$ are shown in Fig. 1a and Fig. 1b. In this study, the KM-function was used in two parametrization forms with a different number of parameters (Tab. 1). The three-parameter form was derived from the four-parameter version by fixing the parameter $t_{\max }$ to a value of 500 years on the basis of the maximum lifetime estimated for beech trees in Slovakia (Pagan 1992).

The Richards' function is one of the most important growth models of the exponential decline type sensu Zeide (1993), and repre- 
sents one of the most used growth models worldwide. Its popularity stems from the excellent flexibility, along with a relatively good extrapolation capability. Similarly to the KM-function, the Richards' function is also used in its forms including three or four parameters.

The Korf's growth function belongs to the power decline type sensu Zeide (1993), and it is considered as the most suited to forestry conditions of Slovakia. It was used for the construction of the national growth and yield tables (Halaj \& Petráš 1998). According to Zeide (1989), this function is especially suitable for the description of the diameter of a fixed number of trees, as it is more accurate than other traditional growth functions.

The Weibull's growth function does not belong to any of the aforementioned classification sensu Zeide (1989). From a practical point of view, this function has the same advantages as the Richards' function, and it was included in the list of Tab. 1 because it was derived exclusively in an empirical way, just as the KM-function.

All the classical growth functions described above share the common feature of being time-non-determinate growth models with an asymptotic behavior.

\section{Dataset}

The empirical dataset used in this study consisted of stem analyses for 67 beech trees cut in an 80-160 years-old mature fores stand growing in a good-quality site. The analysis of the selected growth functions and parametrization methods was performed on growth series at time intervals of five years based on the diameter at breast height $\left(d_{1.3}\right)$ measured to the nearest $1 \mathrm{~mm}$. Current and mean annual increments were inferred from diameter growth data. Growth and incremen curves were subjected to graphical analysis, revealing two episodes of abrupt increases in current increment along the stand history. The first episode was interpreted as due to an intensive thinning carried out prior to the culmination of current increments at the age of 50-70 years, while the other was at the beginning of the natural regeneration step at the age of 120-140 years. From a modeling point of view, the increase of tree growth at later stages due to seeding felling is in contradiction with the sigmoid-shaped growth models, that predict a reduction of current increment at older ages. Therefore, each diameter growth series was truncated, considering only the data prior to the start of the stand's natural regeneration (identified at age 120 years). Moreover, data from trees with age $<20$ years were excluded from growth series, since their diameters were affected by rather high measurement errors. In total, 67 growth series with age ranging from 20 to 115 years were included in the analysis. Since data were grouped in 5 years inter- vals, each individual growth series was composed by 19 measured diameters. More detailed information on individual series and discrete events along the stand history are given in Tab. 2.

\section{Error analysis}

Performances of growth functions were assessed by estimating the bias and accuracy of short-term (5 years) predictions of tree diameter growth. Comparison of observed vs. predicted values for the variables considered represent a commonly-used, simple method to evaluate growth models (Pretzsch 2009, Vanclay 1994). Model validation through the analysis of deviations of projected values from empirical data, allows an objective estimation of the ability of growth functions to capture meaningful biological trends, and extrapolate growth changes over time, thus assessing its suitability to modeling purposes (Ek \& Monserud 1979, Zhang 1997).

Each growth series was divided into two parts for parametrization and validation purposes. The parametrization step was carried out on sequences of 18 measurements at the age ranging from 25 to 110 years covering all the life stages (juvenility, maturity, senescence). The validation step was carried out on one measurement at the age of 115 years. This measurement was omitted from the existing growth series and used for comparison of real diameter at this age with the diameter estimated by forward extrapolation of the parametrization sequences.

Relative errors of extrapolation for tree diameters were calculated at the validation step as $e \%=\left(d_{\mathrm{p}}-d_{\mathrm{r}}\right) / d_{\mathrm{r}} \cdot 100$, where $d_{\mathrm{p}}$ is the predicted diameter and $d_{\mathrm{r}}$ is the real diameter at age 115 years. Every selected growth equation was parametrized for every individual tree. Therefore, a set of 67 individual errors were obtained for every used growth function. The arithmetic mean of individual tree errors $\bar{e} \%$ was calculated as a measure of bias, and the root mean square error (RMSE) as a measure of the absolute size of errors, thus indicating the practical applicability of the function considered.

\section{Parametrization}

As for classic (frequentist) approach, parametrization of growth functions was done using the NLS method implemented in the software package Statistica ${ }^{\circledR}$ v. 10.0 (StatSoft 2010). Among different numerical opti-

Tab. 2 - Main statistics of the empirical growth series analyzed. (SD): Standard deviation; $(\mathrm{dbh})$ : diameter at the breast height.

\begin{tabular}{lcccccc}
\hline Statistics & $\begin{array}{c}\text { Current incre- } \\
\text { ment culmina- } \\
\text { tion (yrs) }\end{array}$ & $\begin{array}{c}\text { Mean incre- } \\
\text { ment culmi- } \\
\text { nation (yrs) }\end{array}$ & $\begin{array}{c}\text { Age of 1 } \\
\text { release } \\
\text { (yrs) }\end{array}$ & $\begin{array}{c}\text { Beginning of } \\
\text { regeneration } \\
\text { (yrs) }\end{array}$ & $\begin{array}{c}\text { Cut down } \\
\text { age (yrs) }\end{array}$ & $\begin{array}{c}\text { Cut down } \\
\text { dbh } \\
\text { (cm) }\end{array}$ \\
\hline Minimum & 27 & 33 & 25 & 75 & 85 & 17.3 \\
Mean \pm SD & $72 \pm 14$ & $95 \pm 20$ & $61 \pm 18$ & $114 \pm 18$ & $131 \pm 17$ & $37.1 \pm 9.7$ \\
Maximum & 100 & 145 & 110 & 150 & 165 & 54.4 \\
\hline
\end{tabular}

mization methods, the derivative LevenbergMarquardt method was primarily chosen; when convergence using this method failed, the more robust non-derivative techniques of direct search, the Hooke-Jeeves and Rosenbrock methods, were applied instead. The simple form of the NLS method was used, despite the inherent heteroscedasticity and autocorrelation of growth residuals. Indeed, autocorrelation and non-homogeneity of the variance of residual deviations do not represent a serious problem for predictions, since parameters estimates of growth models are not biased, as previously reported by several authors (Bock \& du Toit 2004, Vanclay 1994).

Bayesian parametrization starts with the formulation of the a priori probability distributions of individual growth functions parameters. The marginal a priori parameter distribution represent the probability of occurrence of parameter values for the selected growth functions, when modeling of diameter growth is applied to individual beech trees growing in different social positions and in sites of varying quality under natural conditions of Slovakia. Such distributions were mathematically formalized (elicited) by a special mathematical-statistical procedure based on the calculation of percentiles of the empirical diameter distributions (for a detailed description, see Sedmák 2009). Halaj (1957) reported the empirical distribution of the relative frequencies of $2-\mathrm{cm}$ diameter classes in 420 beech stands, according to different stand mean diameters (from 10 to 50 $\mathrm{cm}$, scaled by $2 \mathrm{~cm}$ ), along with the so-called "degree of stand volume variance" (lower, average and higher degree). Such information has been included in the National growth and yield tables (Halaj \& Petráš 1998). By combining the information on age and site class, mean stand diameter may be predicted at any age for different site class, and consequently the percentiles of the corresponding diameter distribution (99 percentile growth series for percentiles 1-100) may be obtained. The percentile growth series simulate the growth in diameter of individual beech trees growing in different social position. Fitting all growth functions to these series using the NLS method, we obtained information on possible values of function parameters for each site class. Consequently, we could infer the probability distribution, mean values, variance and covariance of in- 
Tab. 3 - Error statistics of diameter predictions for trees at the age 115 years according to growth functions and parametrization methods. (NLS): nonlinear least squares; (RMSE): root mean square error; (1): highlighted are minimal values of the statistic within each modeling aim (minimization of bias or RMSE); (2): only absolute value of biases are divided to analyze their magnitude irrespectively of their sign; (3): only absolute value of biases were averaged for the same reason than in (2).

\begin{tabular}{|c|c|c|c|c|c|c|c|}
\hline \multirow{3}{*}{$\begin{array}{l}\text { No. } \\
\text { Parameters }\end{array}$} & \multirow{3}{*}{$\begin{array}{l}\text { Growth } \\
\text { function }\end{array}$} & \multicolumn{6}{|c|}{ Parametrization methods } \\
\hline & & \multicolumn{2}{|c|}{ NLS } & \multicolumn{2}{|c|}{ Bayes } & \multicolumn{2}{|c|}{ Bayes/NLS ratio } \\
\hline & & $\begin{array}{c}\text { RMSE } \\
\%\end{array}$ & $\begin{array}{c}\text { Bias } \\
\%\end{array}$ & $\begin{array}{c}\text { RMSE } \\
\%\end{array}$ & $\begin{array}{c}\text { Bias } \\
\%\end{array}$ & $\begin{array}{c}\text { Ratio of } \\
\text { RMSEs }\end{array}$ & $\begin{array}{l}\text { Ratio of } \\
\text { Biases }^{(2)}\end{array}$ \\
\hline \multirow{4}{*}{$\begin{array}{l}\text { 3-parameter } \\
\text { functions }\end{array}$} & Korf (KF3) & 6.4 & 4.9 & 5.9 & 3.7 & 0.92 & 0.76 \\
\hline & Richards (R3) & 6.8 & 5.0 & 5.5 & 3.9 & 0.81 & 0.77 \\
\hline & KM-function (KM3) & 17.8 & $-1.1^{(1)}$ & $4.3^{(1)}$ & $2.1^{(1)}$ & 0.24 & 1.93 \\
\hline & Average & 10.3 & $3.7^{(3)}$ & 5.3 & 3.2 & 0.66 & 1.15 \\
\hline \multirow{4}{*}{$\begin{array}{l}\text { 4-parameter } \\
\text { functions }\end{array}$} & Weibull (W4) & 3.7 & 2.1 & 6.1 & 3.8 & 1.68 & 1.82 \\
\hline & Richards (R4) & 7.2 & 6.4 & 6.7 & 5.9 & 0.93 & 0.92 \\
\hline & KM-function (KM4) & 8.6 & 7.5 & 19.8 & -17.6 & 2.30 & 2.36 \\
\hline & Average & 6.5 & 5.3 & 10.9 & 9.1 & 1.64 & 1.707 \\
\hline
\end{tabular}

dividual parameters for each selected function. Then, convenient marginal probability distributions of individual parameters were found by Pearson's $\chi^{2}$ test. The elicited marginal a priori distributions of individual parameters had a form of either lognormal or Gamma distribution. Joint a priori distributions of multiple parameters were obtained as the simple product of marginal distributions of individual parameters.

Bayes theorem was applied as a combination of a normal likelihood function with joint a priori distributions determined by elicitation. According to the Bayesian approach, parameter estimates were calculated by the Gibbs sampling method using the software package WinBugs 1.4 (Lunn et al 2000). The Gibbs method belongs to a group of numerical methods of integration of multiple integrals referred to as Markov Chain Monte Carlo (MCMC) methods. To generate the numerical estimates of the parameters for the growth functions to be compared, 11 MCMC chains were used and a posteriori parameter distributions were composed based on numerical generation of about 500000 values. With regard to multimodality of some a posteriori marginal parameter distributions, the final parameter estimates were obtained as the medians of the a poste-

Tab. 4 - Ratios of the RMSE and bias vs. minimal values of the RMSE and bias reported in Tab. 3. (NLS): nonlinear least squares; (RMSE): root mean square error; (1): best ratios according to the modeling aim (minimization of bias or RMSE).

\begin{tabular}{llcccccc}
\hline \multirow{2}{*}{$\begin{array}{l}\text { No. } \\
\text { Parameters }\end{array}$} & \multirow{2}{*}{$\begin{array}{c}\text { Growth } \\
\text { function }\end{array}$} & \multicolumn{6}{c}{ Parametrization methods } \\
\cline { 2 - 8 } & & \multicolumn{2}{c}{ NLS } & \multicolumn{2}{c}{ Bayes } & \multicolumn{2}{c}{ Average } \\
\cline { 2 - 7 } & RMSE & Bias & RMSE & Bias & RMSE & Bias \\
\hline 3-parameter & Korf (KF3) & 1.73 & 4.41 & 1.37 & 1.76 & 1.55 & 3.09 \\
functions & Richards (R3) & 1.84 & 4.50 & 1.28 & 1.86 & 1.56 & 3.18 \\
& KM-function (KM3) & 4.81 & $1.00^{(1)}$ & $1.00^{(1)}$ & $1.00^{(1)}$ & 2.91 & $1.00^{(1)}$ \\
& Average & 2.79 & 3.31 & 1.22 & 1.54 & 2.00 & 2.42 \\
\hline 4-parameter & Weibull (W4) & $1.00^{(1)}$ & 1.89 & 1.42 & 1.81 & $1.21^{(1)}$ & 1.85 \\
functions & Richards (R4) & 1.95 & 5.77 & 1.56 & 2.81 & 1.75 & 4.29 \\
& KM-function (KM4) & 2.32 & 6.76 & 4.60 & 8.38 & 3.46 & 7.57 \\
& Average & 1.76 & 4.80 & 2.53 & 4.33 & 2.14 & 4.57 \\
\hline
\end{tabular}

are rather small (Tab. 3). Most of the RMSE lie within the interval of $3.7-8.6 \%$, with two exceptions approaching the limit of $20 \%$. Similarly, most of biases were ranging 1.1$7.5 \%$, with only one exception reaching $17.6 \%$. Most of the bias signs were positive, indicating a tendency to overestimation of real diameters in the prediction of future growth.

The results of the comparison of parametrization methods regardless of the respective growth function (columns "NLS" and "Bayes" - Tab. 3) showed that the Bayesian parametrization was moderately to significantly better as for RMSE in four out of six growth functions, though in two cases it was significantly worse. From the viewpoint of bias, the situation is more balanced ( $3 \times$ better NLS, $3 \times$ better Bayes). However, in cases where NLS gave better results, its prevalence was pronounced, whereas in cases where better results were obtained using the Bayesian approach, its prevalence was only moderate.

Regardless the parametrization method, the analysis of growth function efficiency according to a number of parameters (columns and lines "Average"- Tab. 4) showed that three-parameter functions perform better for both aims of modeling. Differences in mean RMSE ratios averaged across functions and parametrization methods are much smaller than in mean bias ratios. This means that three-parameter functions performed only slightly better for error magnitude, but their predictions are clearly less biased; therefore, three-parameter functions remarkably produced more realistic predictions.

A comparison of the function categories (three or four-parameter) within a specific parametrization methods (Tab. 4, columns "NLS" and "Bayes", lines "Average") showed that three-parameter functions performed much better for both modeling aims within Bayesian parametrization and were also less biased when the NLS parametrization method is used. Four-parameter functions are much more suitable for a minimization of RMSE within NLS, although they are somewhat more biased.

A more detailed comparison of the most successful combinations of function category and methods (three-parameter growth functions/Bayes parametrization and four-parameter growth functions/NLS) led to the conclusion that three-parameter growth functions combined with the Bayesian parametrization method gave better results for both modeling aims. The combination of three-parameter growth functions with the Bayesian approach (column "Bayes" - Tab. 3) showed a smaller RMSE by $15-25 \%$ as well as smaller biases by $40-60 \%$ than fourparameter functions combined with the NLS parametrization method (column "NLS" Tab. 3). 
Comparison of individual growth functions regardless of the parametrization method showed that the Weibull (W4) and the KM3 functions were the most successful. The W4 function gave better results from the point of view of RMSE, while the KM3 function performed better from the point of view of bias (columns "Average" - Tab. 4). It is worth to notice that the KM3 function produced better results in combination with the Bayesian parametrization, while the $\mathrm{W} 4$ function in combination with the use of the NLS method. Also, the KM3 function gave three times better results for a various combinations of modeling aims and parametrization methods (Tab. 4), in particular, the KM3 function was the best for combinations bias/ NLS, bias/Bayes and RMSE/Bayes (Fig. 2).

Comparing the selected growth functions across parametrization methods, the W4 function produced more robust results and smaller differences among parametrization methods. Moreover, the KM3 function can produce excellent results as far as biases are concerned. However, when using the NLS method, the small bias was offset by a significant decrease in the overall accuracy of predictions. Furthermore, the four-parameter version of the KM-function resulted among the worst functions, especially in combination with the Bayes parametrization. This evidence is exactly the opposite of that obtained from the results of three-parameter version. Similar considerations hold for the three and four-parameter versions of the Richards function. In the latter case, the three-parameter function was similarly better than the four-parameter version for both parametrization methods and modeling aims, although differences were not so pronounced.

\section{Discussion}

The most important characteristic of the $\mathrm{KM}$-function is its time-determinate feature, i.e., the function predicts the complete termination of growth when the organism reaches its final size. A more detailed analysis of the integral and differential forms of the KMfunction showed that:

1. The integral form (eqn. 2) admits the possibility of starting the growth at the age of $t(0)=0$, and at a given age, it simultaneous ly admits the possibility $y(0)=0$. The differential form (eqn. 3) at the age of $t(0)=0$ predicts $d y / d t=0$; RGR at the age of $t(0)=0$ is not defined. However, it holds true that if $t(0) \rightarrow 0$, RGR is limited in its approach to infinite $\mathrm{RGR}_{\max }$.

2. The position of the inflexion point within the life cycle is not fixed. The inflexion of the growth curve can occur at any age $t$ and value $y$.

3. In the period of growth cessation at the time $t=t_{\max }$, the integral form (eqn. 2) predicts $y=y_{\max }$, and simultaneously (eqn. 3) at
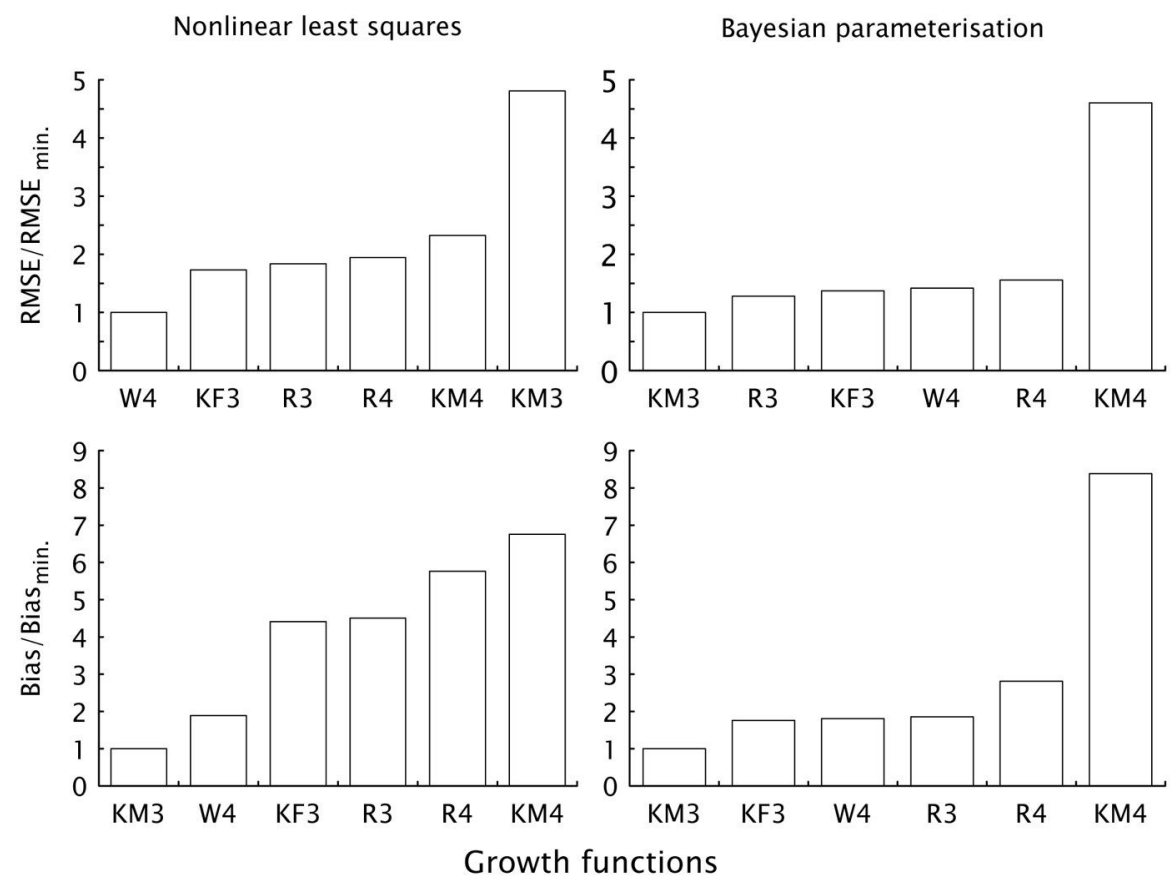

Fig. 2 - Ranking of the analyzed growth functions according to the modeling aim (minimization of bias or RMSE) and the parametrization method used (NLS or Bayesian).

the age of $t=t_{\max }$ it holds true that $d y / d t=0$ and consequently, RGR $=0$.

Property (1) is shared by Richards, Weibull and KM functions and contributed significantly to their popularity. It does not contradict the known biological laws and contributes to the feasibility of growth modeling at the early life stages. The Korf function at the age of $t(0)=0$ has neither the defined value $y(0)$ nor the value $d y / d t$, thus the possibility for growth to start from the zero point is excluded.

From properties $(2,3)$ it follows that the $\mathrm{KM}$-function is a model of time-determinate asymmetrical growth starting at zero size of an organism and terminating when it reaches its final size. Since the KM-function does not merely approach asymptotically, but exactly reaches both the starting and the final size, the equation assumes a finite length for the growth period of an organism. This represents an independent parameter in the equation $t_{\max }$ and can be satisfactorily estimated by means of the maximum age of the tree species under the particular natural conditions. Simultaneously, the age of growth rate culmination can occur at any time $t \in[0$, $\left.t_{\max }\right]$ and at any value $y$, resulting in the capability of the growth function to represent any degree of time growth asymmetry.

The growth function Beta (Yin et al. 2003) is the only other function known from the literature which is able to describe in a continuous way the determinate growth of living organisms. It is based on the application of the frequency function of Beta distribution, which is a more general alternative to the
KM-distribution. As a result, a number of common properties are shared by the Beta and the KM-function.

Yin et al. (2003) point out the following properties of the Beta function: (i) its flexibility - the point of inflexion of the growth trajectory may occur at any position; (ii) good parametrization properties resulting from clear interpretability of parameters, and in particular (iii) the possibility to estimate the final value of yield at the end of the production period. These advantages are also true for the KM-function, especially in its three-parameter version.

Easy and stable parametrization of the three-parameter KM-function results from a good biological interpretability of parameters, likely stemming from its smaller intrinsic curvature. Indeed, the integral form of the KM-function does not contain any exponential terms (eqn. 2). Consequently, the equation is similar to Hossfeld and Levaković growth functions (Zeide 1993 - eqn. 4, eqn. 5):

$$
\begin{array}{ll}
\text { Hossfeld: } & y=\frac{t^{c}}{\left(b+t^{c}\right) / a} \\
\text { Levakovic: } & y=a t\left(\frac{t^{d}}{b+t^{d}}\right)^{c}
\end{array}
$$

According to Kiviste (1988), Zeide (1993) reports that these equations are surprisingly accurate, though they are among the oldest growth functions known.

On the other hand, the four-parameter KM function version provided poor predictions on the dataset tested in this study. Also, the 
three-parameter KM function along with NLS parametrization method provided relatively large RMSE. The above facts suggest that, despite its expected small intrinsic curvature, the KM function may have a rather large parameter-effect curvature. The inclusion of an additional parameter into the KM function led to the instability in the estimation of parameters, and consequently the accuracy was notably reduced, even for shortterm predictions. The same scenario hold also for the Richards' function.

The problem of the relatively large parameter-effect curvature of the KM-function may be solved by the inclusion of biologically meaningful constraints into the process of parameter estimation. For example, fixing the parameter value $t_{\max }$ based on its biological interpretation (i.e., the expected lifespan of the organism considered) has proven to be useful, especially when further information on other parameters was included into the estimation process by the Bayesian estimation procedure. As a result, the three-parameters KM function was assessed as the second most successful function.

A good accuracy of the KM-function is also expected based on additional theoretical reasons. Despite several shared properties, $\mathrm{KM}$ and Beta functions also show some differences. The differential form of the Beta function, obtained from the original frequency function of the Beta distribution under the assumption $t(0)=0$ and arbitrarily fixing the parameter $\delta=1$, is the following (Yin et al. 2003 - eqn. 6):

$$
\frac{d y}{d t}=c\left(\frac{t_{\max }-t}{t_{\max }-t_{i}}\right)\left(\frac{t}{t_{i}}\right) \frac{t_{i}}{t_{\max }-t_{i}}
$$

where $t_{\mathrm{i}}$ is the time of growth rate culmination and $c$ is the maximum growth rate at time $t_{\mathrm{i}}$. The above function has both expansion and restriction elements linked to age, i.e., the expansion element is a power function and the restriction element is a linear function of age. The biological rationale for the linearity of the Beta function restriction element is problematic: the problem originates from the fixation of $\delta$ in the original Beta function because of the need for analytical integrability of the eqn. 6 . In the KMfunction, both elements represent a power function of age, thus being theoretically better fitting to empirical data.

Linkage to $t$ of expansion and restriction elements of the differential form is a quite rare property. The age $t$ has both positive and negative influence on growth rate $d y / d t$. though the negative influence prevails increasing the age. The only other well-established function having this property is the Weibull growth function, whose differential form is as follows (eqn. 7):

$$
\frac{d y}{d t}=k t^{p} e^{-q t^{-c}}
$$

Another similar growth function was proposed by Sloboda (eqn. 8):

$$
\frac{d y}{d t}=k y t^{d-1} e^{-b t^{d}}
$$

whose expansion element is linked to the interaction of age and size. In almost all other growth functions, the expansion element is linked to size $y$ and the restriction component to age $t$. Consequently, KM, Weibull and Sloboda's functions admit the possibility of initial age $t(0)=0$, assuming $d y / d t=0$ at this age. On the other hand, classical Weibull and Sloboda's equations are typical representatives of non-determinate growth models, in which if $t \rightarrow \infty$, then $y \rightarrow y_{\max }$ and $d y / d t \rightarrow 0$, i.e., they implicitly assume an infinite lifespan for the organism studied.

The KM, Beta and Weibull growth functions, along with the Richards and Korf functions, share an additional feature: all functions have $y(0)=0$ at the age $t(0)=0$, that is, they do not have a defined RGR (i.e., if $t \rightarrow 0$ then RGR $\rightarrow \infty$ ). Unlike the above equations, the integral form of the Sloboda's model does not allow the possibility $y=0$ at any age, thus RGR is defined and equal to 0 at the age $t(0)=0$. Based on the above considerations, it follows that Weibull, Beta and KM functions are not suitable for modeling the exponential growth in the initial life stages (e.g., at $t<1$ ), since the classical growth analysis assumes that the growth rate is proportional to size, i.e., the final RGR approaches the final maximum value.

Comparison of the selected growth function properties proved the usefulness of the $\mathrm{KM}$-function for growth modeling purposes. The three-parameter version (KM3), combined with the Bayes parametrization method, was the second most successful function for short-term predictions of tree diameter growth on the studied dataset. In particular, such model was suitable for bias minimization.

As expected, results of this study showed that the Bayesian parametrization method performs slightly better in minimizing the modeling errors. Also, NLS was slightly more suitable when minimization of biases is required in short-term predictions. However, differences in RMSE and biases among the parametrization methods used were relatively small, probably due to the fairly large number of measurements covering most of the life cycle of trees. Using high quality empirical measurements, the above differences are diminished because the importance of $a$ priori parameter distributions quickly decreases in the Bayesian procedure (Bock \& du Toit 2004).

\section{Conclusions}

The analysis carried out revealed several useful theoretical properties of the KM-function:
- good fitting to empirical data and high interpolation accuracy, due to the possibility of inflexion at any $y$ value and the power form of both key components in the differential equation;

- realistic growth reconstruction of backward extrapolations based on empirical measures, thanks to the possibility of predicting $y(0)=0$ and $d y / d t=0$ at the age $t(0)=0$, consistently with known biological growth laws.

- realistic growth predictions of forward extrapolations based on empirical measures, thanks to the a priori inclusion of a parameter related to the final length of life cycle and the final size of the organism.

Results of the comparison of the KM-function with selected classical growth functions proved its usefulness for modeling the growth in diameter of individual trees, in particular in its three-parameter form combined with Bayesian parametrization when minimization of prediction bias is needed, while the use of four-parameter Weibull function combined with the NLS parametrization method is recommended for the short-term prediction of diameter growth.

\section{Acknowledgements}

This study was supported by the European Structural Fund under the project ITMS: 26220120069 (Center of Excellence for decision support in forest and landscape at the Technical University in Zvolen, Slovakia, Activity 3.1 Experimental and methodological platform of precision forestry tools) and by the Scientific Grant Agency of the Ministry of Education, Science, Research and Sport of the Slovak Republic under the project $1 / 0618 / 12$ and $1 / 0953 / 13$. Additional support was received from the National Agency of Agricultural Research of the Czech Republic under the contract No. QJ1320230.

\section{References}

Bertalanffy L (1957). Quantitative laws in metabolism and growth. Quarterly Review of Biology 32: 217-231. - doi: 10.1086/401873

Burkhart HE, Tomé M (2012). Modelling forest trees and stands. Springer Science + Business Media BV, Dordrecht, The Netherlands, pp. 471. [online] URL: http://books.google.com/books? id $=$ XmcNcfEarsoC

Birch CP (1999). A new generalized logistic sigmoid growth equation compared with the $\mathrm{Ri}$ chards growth equation. Annals of Botany 83: 713-723. - doi: 10.1006/anbo.1999.0877

Bock RD, du Toit SHC (2004). Parameter estimation in the context of nonlinear longitudinal growth models. In: "Methods in Human Growth Research" (Hauspie RC, Cameron N, Molinari L eds). Series Cambridge Studies in Biological and Evolutionary Anthropology, vol. 39, Cambridge University Press, Cambridge, UK, pp. 198-220.

Carlin BP, Louis TA (2000). Bayes and empirical 
Bayes methods for data analysis. Texts in Statistical Science, Chapman \& Hall/CRC, Boca Raton, FL, USA, pp. 440.

D'Agostini G (2003). Bayesian inference in processing experimental data: principles and basic applications. Reports on Progress in Physics 66 (9): 1383-1383. - doi: 10.1088/0034-4885/66/9/ 201

Ek AR, Monserud RA (1979). Performance and comparison of stand growth models based on individual tree diameter. Canadian Journal of Forest Research 9: 231-244. - doi: 10.1139/x79-040

Fekedulegn D, Mac Siurtain MP, Colbert JJ (1999). Parameter estimation of nonlinear growth models in forestry. Silva Fennica 33 (4) 327-336. - doi: 10.14214/sf.653

Fitzhugh HA (1976). Analysis of growth curves and strategies for altering their shape. Journal of Animal Science 42 (4): 1036-1051. [online] URL: http://www.journalofanimalscience.org/ content/42/4/1036.short

Gompertz B (1825). On the nature of the function expressive of the law of human mortality, and on a new mode of determining the value of life contingencies. Philosophical Transactions of the Royal Society 115: 513-585. - doi: 10.1098/rstl. 1825.0026

Halaj J, Petráš R (1998). A growth and yield tables of main tree species in Slovakia. Slovak Academic Press, Bratislava, SK, pp. 325. [in Slovak]

Halaj J (1957). Mathematical and statistical research of diameter structures of Slovak stands. Lesnícky časopis 3 (1): 39-74. [in Slovak]

Karkach AS (2006). Trajectories and models of individual growth. Demographic Research 15: 347-400. - doi: 10.4054/DemRes.2006.15.12

Kiviste AK (1988). Mathematical functions of forest growth. Estonian Agriculture Academy, Tartu, Estonia, pp.108.

Korf V (1939). Contribution to mathematical definition of the law of stand volume growth. Manuscript, Lesnická práce, Slovakia, pp. 339-379.

Kumaraswamy P (1980). A generalized probability density function for double-bounded random processes. Journal of Hydrology 46: 79-88. - doi: 10.1016/0022-1694(80)90036-0
Li FG, Zhao BD, Su GL (2000). A derivation of the generalized Korf growth equation and its application. Journal of Forestry Research 11 (2): 81-88. - doi: 10.1007/BF02856679

Lunn DJ, Thomas A, Best N, Spiegelhalter D (2000). WinBUGS - a Bayesian modeling framework: concepts, structure, and extensibility. Statistics and Computing 10: 325-337. - doi: 10.10 23/A:1008929526011

Mitscherlich EA (1919). Problems of plant growth. Landwirtschaftliche Jahrbücher 53: 167182. [in German]

Pagan J (1992). Forestry dendrology. Technical University in Zvolen, Zvolen, Slovakia, pp. 347. [in Slovak]

Pretzsch H (2009). Forest dynamics, growth and yield. From measurement to model. SpringerVerlag, Berlin, Germany, pp. 617. - doi: 10.1007 /978-3-540-88307-4

Ratkowsky DA (1983). Nonlinear regression modelling. Marcel Dekker, New York, USA, pp. 276.

Richards FJ (1959). A flexible growth function for empirical use. Journal of Experimental Botany 10: 290-300. - doi: 10.1093/jxb/10.2.290

Seber GAF, Wild CJ (2003). Nonlinear regression. Wiley series in probability and statistics, John Wiley \& Sons, Inc., New Jersey, USA, pp. 792.

Sedmák R (2009). Growth and yield modelling of beech trees and stands. Msc Thesis, Technical University in Zvolen, Zvolen, Slovakia, pp. 181.

Sedmák R, Scheer L (2012). Modelling of tree diameter growth using growth functions parameterised by least squares and Bayesian methods. Journal of Forest Science 58 (6): 245-252. [online] URL: http://www.agriculturejournals.cz/ publicFiles/66810.pdf

Shvets V, Zeide B (1996). Investigating parameters of growth equations. Canadian Journal of Forest Research 26: 1980-1990. - doi: 10.1139/ x26-224

Sloboda B (1971). Investigation of growth processes using first order differential equations. Mitteilungen der Baden-Württembergischen Forstlichen Versuchs und Forschungsanstalt 32: 1-109. [in German]

Schnute J (1981). A versatile growth model with statistically stable parameters. Canadian Journal of Fisheries and Aquatic Sciences 38: 11281140. - doi: 10.1139/f81-153

StatSoft Inc (2010). Electronic statistics textbook. Tulsa, OK, USA. [online] URL: http://www.statsoft.com/textbook

Tsoularis A, Wallace J (2002). Analysis of logistic growth models. Mathematical Biosciences 179: 21-25. - doi: 10.1016/S0025-5564(02)00096-2

Vanclay JK (1994). Modelling forest growth and yield: application to mixed tropical forests. $\mathrm{CAB}$ International, Wallingford, UK, pp. 312. [online] URL: http://epubs.scu.edu.au/cgi/viewcontent.c gi? article $=1538 \&$ context $=$ esm_pubs

Verhulst B (1838). A note on population growth. Correspondence Mathematiques et Physiques 10 113-121. [in French]

Weibull W (1951). A statistical distribution of wide applicability. Journal of Applied Mechanics 18: 293-297.

Weiskittel AR, Hann DW, Kershaw JA, Vanclay JK (2011). Forest growth and yield modelling (1st edn). John Wiley \& Sons Ltd, Chichester, UK, pp.344. [online] URL: http://books.google.com/books?id=MJ4_S8srXx4C

Yin X, Goudriaan J, Lantinga EA, Vos J, Spiertz HJ (2003). A flexible sigmoid function of determinate growth. Annals of Botany 91 (3): 361371. - doi: 10.1093/aob/mcg029

Zeide B (1989). Accuracy of equations describing diameter growth. Canadian Journal of Forest Research 19 (10): 1283-1286. - doi: 10.1139/x89195

Zeide B (1993). Analysis of growth equations. Forest Science 39 (3): 594-616. [online] URL: http://www.ingentaconnect.com/content/saf/fs/1 993/00000039/00000003/art00014

Zeide B (2003). The U-approach to forest modelling. Canadian Journal of Forest Research 33 (3): 480-489. - doi: 10.1139/x02-175

Zeide B (2004). Intrinsic units in growth modelling. Ecological Modelling 175 (3): 249-259. doi: 10.1016/j.ecolmodel.2003.10.017

Zhang L (1997). Cross-validation of non-linear growth functions for modelling tree height-diameter relationships. Annals of Botany 79 (3): 251-257. - doi: 10.1006/anbo.1996.0334 